\title{
Recombinant Human Serum Albumin Dimer has High Blood Circulation Activity and Low Vascular Permeability in Comparison with Native Human Serum Albumin
}

\author{
Sadaharu Matsushita, ${ }^{1}$ Victor Tuan Giam Chuang, ${ }^{1,2}$ Masanori Kanazawa, ${ }^{1}$ Sumio Tanase, ${ }^{3}$ \\ Keiichi Kawai, ${ }^{4}$ Toru Maruyama, ${ }^{1}$ Ayaka Suenaga, ${ }^{1}$ and Masaki Otagiri ${ }^{1,5}$
}

\begin{abstract}
Received September 8, 2005; accepted January 12, 2006
Purpose. Human serum albumin (HSA) is used clinically as an important plasma expander. Albumin infusion is not recommended for critically ill patients with hypovolemia, burns, or hypoalbuminemia because of the increased leakage of albumin into the extravascular spaces, thereby worsening edema. In the present study, we attempted to overcome this problem by producing a recombinant HSA (rHSA) dimer with decreased vascular permeability and an increased half-life.

Methods. Two molecules of rHSA were genetically fused to produce a recombinant albumin dimer molecule. The pharmacokinetics and biodistribution of the recombinant proteins were evaluated in normal rats and carrageenin-induced paw edema mouse model.

Results. The conformational properties of this rHSA dimer were similar to those for the native HSA (the HSA monomer), as evidenced by the Western blot and spectroscopic studies. The biological halflife and area under the plasma concentration-time curve of the rHSA dimer were approximately 1.5 times greater than those of the monomer. Dimerization has also caused a significant decrease in the total body clearance and distribution volume at the steady state of the native HSA. rHSA dimer accumulated to a lesser extent in the liver, skin, muscle, and fat, as compared with the native HSA. Up to $96 \mathrm{~h}$, the vascular permeability of the rHSA dimer was less than that of the native HSA in paw edema mouse models. A prolonged plasma half-life of the rHSA dimer was also observed in the edema model rats. Conclusions. rHSA dimer has a high retention rate in circulating blood and a lower vascular permeability than that of the native HSA.
\end{abstract}

KEY WORDS: biodistribution; human serum albumin; pharmacokinetic analysis; recombinant human serum albumin dimer; vascular permeability.

\section{INTRODUCTION}

Human serum albumin (HSA), the most abundant protein in plasma, is responsible for $80 \%$ of the colloid osmotic pressure of plasma $(25-33 \mathrm{mmHg})$. Because of this, it

\footnotetext{
${ }^{1}$ Department of Biopharmaceutics, Graduate School of Pharmaceutical Sciences, Kumamoto University, 5-1 Oe-honmachi, Kumamoto 862-0973, Japan.

${ }^{2}$ Department of Pharmacy, Faculty of Allied Health Sciences, Universiti Kebangsaan Malaysia, Jalan Raja Muda Abdul Aziz, 50300 Kuala Lumpur Malaysia.

${ }^{3}$ Department of Analytical Biochemistry, School of Health Sciences, Kumamoto University, 4-24-1 Kuhonji, Kumamoto 862-0976, Japan.

${ }^{4}$ School of Health Sciences, Faculty of Medicine, Kanazawa University, 5-11-80 Kodatsuno, Kanazawa 920-1192, Japan.

${ }^{5}$ To whom correspondence should be addressed. (e-mail: otagirim@ gpo.kumamoto-u.ac.jp

ABBREVIATIONS: AUC, area under the plasma concentration-time curve; $\mathrm{CL}_{\text {tot }}$, total body clearance; HSA, human serum albumin; rHSA, recombinant $\mathrm{HAS}$; $\mathrm{Vd}_{\mathrm{ss}}$, distribution volume of the steady state; \% ID, percentage of injected dose.
}

is mainly used clinically to maintain colloid oncotic pressure and to increase the circulating plasma volume (1). HSA product was developed for the treatment of shock where HSA Fraction V, USP, served as an attractive agent for the correction of hypovolemia, burns, and various diseases that result in albumin loss. In spite of the many theoretical benefits of albumin infusion in critically ill patients, there has been little evidence to support its widespread clinical use. For many years, albumin infusion was believed to improve vital prognosis. Recently, a meta-analysis that included 24 studies involving 1419 patients published by Cochran Injuries Group Albumin Reviewers suggested that the administration of albumin-containing fluids resulted to a 1.68 times increase in the relative risk of death when compared to a crystalloid solution (2). In contrast, no difference in mortality was reported in a large-scale clinical trial in Australia and New Zealand, in which $4 \%$ albumin was compared to $0.9 \%$ sodium chloride for intravascular fluid resuscitation in patients in intensive care units (ICUs) (3).

Reviews published on the parenteral use of albumin and the consensus of several conferences recommended that the administration of HSA can be justified for acute circulatory 
problems caused by hypovolemia, but it is seldom warranted and is used merely to increase albumin concentrations in cases of hypoalbuminemia (4). Only shock due to recent blood loss is an indication of the use of parenteral albumin, because the incidence of shock to acute-phase or hyperallergic reactions is accompanied by increased albumin flux to the extravascular compartment and adding to the albumin pool in this case would only expand this compartment and bring on edema (5-7). The lung is particularly susceptible to albumin overloading, and albumin administration for respiratory distress has been a matter of debate because of the possibility that pulmonary edema may result $(8,9)$. Cardiac failure is another hazard of an overzealous increase in circulatory volume (10).

It is well known that capillary protein permeability is increased in many pathological and physiological conditions. For instance, the rate of protein extravasation may increase up to 100 times the normal rate in burn injury. Under such conditions, the albumin molecules administered as a plasma expander are transported to organs and cause a further accumulation of fluids, hence worsening the disease. The frequent administration of albumin infusion to maintain the desired blood concentration, as well as the oncotic pressure, inevitably leads to the poor retention of albumin infused during the disease state. Although albumin receptors have been reported to mediate the transport of albumin across the cellular membrane, the mechanism of leakage during inflammation may involve a different route of extravasation since cytokines are known to loosen tight junctions (11).

In other words, if a form of albumin that possesses good blood retention and low extravasation properties could be developed, the frequency of administration could be decreased and further accumulation of fluids in tissues could be prevented. Hence, increasing the molecular size of albumin to stop leakage to extravascular spaces may be a potential solution in making albumin a superior candidate as a volume replacement therapeutic agent. This type of albumin would not only be superior for clinical use but would also be useful pharmacoeconomically.

In addition, since albumin is typically harvested using conventional techniques involving the fractionation of plasma obtained from blood donors, possible viral/prion contaminants contained in the preparation impose risk to users. As a solution to this problem, the development of recombinant technology in producing therapeutic proteins such as HSA has been promoted. In this study, we genetically fused two molecules of HSA and used Pichia pastoris to express the recombinant HSA (rHSA) dimer protein. The pharmacokinetic and pharmacological properties of the rHSA dimer were then evaluated.

\section{MATERIALS AND METHODS}

\section{Materials}

Native HSA was donated by the Chemo-Sera-Therapeutic Research Institute (Kumamoto, Japan) and defatted by means of a charcoal treatment as described by Chen (12). A chimeric plasmid (pJDB-ADH-L10-HAS-A) having cDNA for the mature form of HSA along with an L10 leader sequence was a gift from Tonen Co. (Tokyo, Japan). The restriction enzymes EcoRI, XhoI, AvaI, BamHI, and SalI and E. coli JM109 were obtained from Takara Shuzo Co., Ltd. (Kyoto, Japan). KOD-Plus-DNA polymerase was obtained from Toyobo Co., Ltd. (Osaka, Japan). The restriction enzyme $P c i$ I was obtained from New England Biolabs (Beverly, MA, USA). A DNA sequence kit was obtained from Perkin-Elmer Applied Biosystems (Foster City, CA, USA). The Pichia Expression Kit and the restriction enzyme NcoI were purchased from Invitrogen Corp. (Carlsbad, CA, USA). [ $\left.{ }^{125} \mathrm{I}\right]$ Iodine-125 was purchased from Amersham Biosciences Corp. (Piscataway, NJ, USA). $\left[{ }^{111} \mathrm{In}\right]$ Indium chloride was supplied by Nihon Medi-Physics (Takarazuka, Japan). Trichloroacetic acid (TCA) was purchased from Nakalai Tesque (Kyoto, Japan). $\lambda$ Carrageenin was purchased from Sigma (St. Louis, MO, USA). Other chemicals used were obtained from commercial suppliers.

\section{Synthesis, Purification, and Structure Analysis of rHSA Dimer}

An rHSA dimer in which two HSA molecules are linked with an amino acid linker (GGGGS) 2 was designed. The rHSA dimer expression vector was constructed through the four-piece ligation of the following: (1) pPIC9K vector was digested with BamHI and EcoRI; (2) the fragment derived from the digestion of pPIC9K with BamHI and XhoI restriction sites was amplified by PCR; (3) the PCR product on the $5^{\prime}$ side of the rHSA dimer, amplified using HSA cDNA as the template, was digested with $X h o \mathrm{I}$ and $A v a \mathrm{I}$ (HSAcDNA-1); (4) the PCR product on the $3^{\prime}$ side of rHSA dimer, which was amplified using HSA cDNA as the template, was digested with $A v a \mathrm{I}$ and EcoRI (HSAcDNA2). These four DNA fragments were separated from the cleavage products by agarose gel electrophoresis and purified.

Construction of the expression vector of the rHSA dimer was achieved by a four-piece ligation (Fig. 1). The resulting vector was transformed into $E$. coli JM109 and amplified. The sequence of the rHSA dimer in the resulting vector was confirmed by verifying the sequence of gel extract cDNA fragments of the resulting vector digested by PciI or NcoI. The resulting vector was introduced into the yeast $P$. pastoris (strain GS115), and the rHSA dimer was expressed and purified. The purified rHSA dimer was confirmed by $\mathrm{N}$ terminal amino acid sequences analysis, sodium dodecyl sulfate polyacrylamide gel electrophoresis (SDS-PAGE), and Western blot analysis. The structure of the rHSA dimer was estimated from fluorescence and circular dichroism (CD) spectral data (13).

\section{Transformation, Expression, and Purification of the rHSA Dimer}

Construction of the expression vector of the rHSA dimer was made by a four-piece ligation (Fig. 1). The resulting vector was transformed into E. coli JM109 and amplified. The sequence of the rHSA dimer in the resulting vector was confirmed on gel extracts of the cDNA fragments of the resulting vector digested by PciI or NcoI. The resulting vector was linearized with $S a l I$ and introduced into the yeast 
A

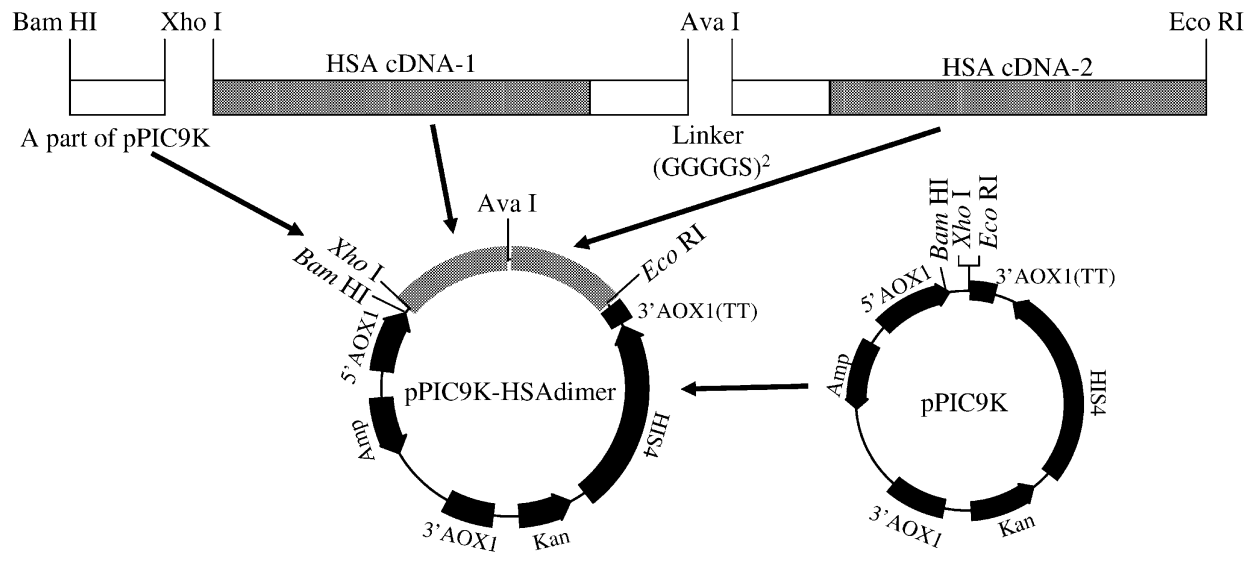

A part of pPIC9K, sense: 5'-CGA AGG ATC CAA ACG ATG AG-3'

antisense: 5'-CTT TTC TCG AGA GAT ACC CCT TCT TC-3'

Xho I

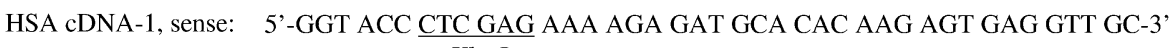

Xho I

antisense: 5'-CAG CTG CC CGA GCC ACC ACC ACC TAA GCC TAA GGC AGC TTG ACT TGC-3' Ava I

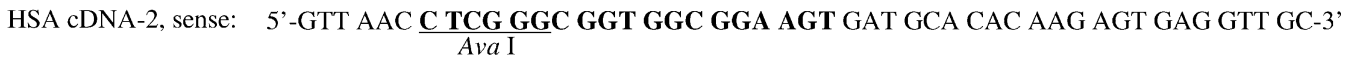

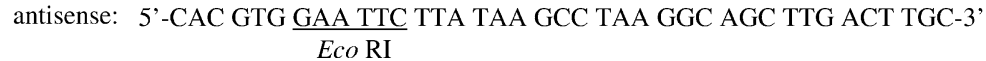

Fig. 1. Schematic of the pPIC9K expression vector containing the rHSA dimer gene (A) and the sequence of the primers used in the construction of the rHSA dimer (B). The restriction site sequences are underlined. The linker regions are in boldface.

$P$. pastoris (strain GS115) by electroporation using a GENEPULSER II (Bio-Rad, Hercules, CA, USA) according to the manufacturer's instruction from Invitrogen. $\mathrm{His}^{+}$colonies were subcultured in yeast protein dextrose (YPD) and plated on YPD agars containing $0.25-3 \mathrm{mg} / \mathrm{mL}$ to select for multicopy recombinants. A $1-\mathrm{mL}$ overnight preculture of the best expression recombinant yeast was used to inoculate $1 \mathrm{~L}$ of glycerol complex medium (1\% yeast extract, $2 \%$ peptone, $100 \mathrm{mM}$ potassium phosphate buffer, $\mathrm{pH} 6.0,1.34 \%$ yeast nitrogen base, $4 \times 10^{-5} \%$ biotin, $3 \%$ glycerol) and incubated for 2 days at $30^{\circ} \mathrm{C}$ in three 3 -L baffle flasks. The cells were harvested by centrifugation $(1500 \times g, 10 \mathrm{~min})$ and resuspended in a buffered $250-\mathrm{mL}$ methanol complex medium $(1 \%$ yeast extract, $1 \%$ peptone, $100 \mathrm{mM}$ potassium phosphate buffer, $\mathrm{pH} 6.0,1.34 \%$ yeast nitrogen base, $4 \times 10^{-5} \%$ biotin, $1 \%$ methanol) for 4 days at $30^{\circ} \mathrm{C}$ in twelve 1 -L baffle flasks. An additional 1\% methanol was added at 12-h intervals. The secreted rHSA dimer was purified on a Blue Sepharose CL-6B column (Amersham Biosciences). Isolated protein was defatted using the charcoal procedure described by Chen (12), deionized, freeze dried, and then stored at $-20^{\circ} \mathrm{C}$ until use. N-terminal amino acid sequences of rHSA dimer were determined using a Perkin-Elmer ABI 477A protein sequencer.

\section{Fluorescence and CD Spectra Measurements}

Intrinsic fluorescence spectra of the proteins were measured using a Jasco FP-777 spectrofluorometer (Tokyo,
Japan) equipped with thermostatically controlled 1-cm quartz cells and 5-nm excitation and emission bandwidths. Native HSA and the rHSA dimer were excited at $295 \mathrm{~nm}$, and the spectra were corrected for buffer baseline fluorescence. CD spectra were obtained using a JASCO J-720 spectropolarimeter (JASCO, Tokyo, Japan) at $25^{\circ} \mathrm{C}$. Farand near-UV CD spectra were recorded at protein concentrations of $5 \mu \mathrm{M}$ (native HSA) and $2.5 \mu \mathrm{M}$ (rHSA dimer) and at $15 \mu \mathrm{M}$ (native HSA) and $7.5 \mu \mathrm{M}$ (rHSA dimer), respectively, in $67 \mathrm{mM}$ phosphate buffer ( $\mathrm{pH} 7.4)$.

\section{SDS-PAGE and Western Blot Analysis}

The rHSA dimer was analyzed via SDS-PAGE, using $8 \%$ polyacrylamide gel, and detected by staining with Coomassie blue. Western blotting was performed using an anti-HSA antibody raised in a rabbit as the primary antibody followed by an antirabbit secondary antibody conjugated to horseradish peroxidase. Proteins were detected using an enhanced chemiluminescence detection kit.

\section{In Vivo Experiments: Animals}

Male Wistar rats (5 weeks old, 150-170 g) and male ddY mice (6 weeks old, 28-30 g) were purchased from the Kyudou Co. (Kumamoto, Japan) and Japan SLC, Inc. (Shizuoka, Japan), respectively. The animals were maintained under conventional housing conditions, with free access to water and 
food throughout the experimental period, and allowed to acclimatize to the laboratory environment for 1 week. After 1 week (1 week before injection of labeled protein), rats injected with ${ }^{111}$ In-labeled protein were given water, which contains $5 \mathrm{mM}$ sodium iodide, until the end of the experiment. All animal experiments were undertaken according to the guideline principles and procedures of Kumamoto University for the care and use of laboratory animals.

\section{Radiolabeling with ${ }^{125}$ I}

Native HSA and rHSA dimer were labeled with ${ }^{125} \mathrm{I}$ using the Iodo-gen (1,3,4,6-tetrachoro-3a,6a-diphenylglycoluri) method (14). Nonincorporated ${ }^{125} \mathrm{I}$ was removed using a PD-10 column (Amersham Biosciences). ${ }^{125}$ I-labeled protein was eluted from PD-10 column with phosphate-buffered saline (PBS). The derivative solution was concentrated and washed with saline by ultrafiltration.

\section{Radiolabeling with ${ }^{111}$ In}

For the tissue distribution experiments, native HSA and rHSA dimer were radiolabeled with ${ }^{111}$ In using the bifunctional chelating agent diethylenetriaminepentaacetic acid (DTPA) anhydride according to the method of Hnatowich et al. (15). In a typical experiment, each sample (5 mg) was dissolved in $1 \mathrm{~mL} 0.1 \mathrm{M}$ 4-(2-Hydroxyethyl)-1piperazineethanesulfonic acid (HEPES) buffer, $\mathrm{pH} 7$, and mixed with $15 \mathrm{mM}$ DTPA anhydride in $10 \mu \mathrm{L}$ dimethyl sulfoxide. The mixture was stirred for $15 \mathrm{~min}$ at room temperature, and the radiolabeled product was purified by gel filtration using a Sephadex G-25 column (Amersham Biosciences) to remove unreacted DTPA. Fractions containing the sample were collected and concentrated by ultrafiltration at $4^{\circ} \mathrm{C}$. A $20-\mu \mathrm{L}$ aliquot of an ${ }^{111} \mathrm{InCl}_{3}$ solution $(37 \mathrm{MBq} / \mathrm{mL})$ was then added to $20 \mu \mathrm{L}$ of $0.1 \mathrm{M}$ citrate buffer, $\mathrm{pH} 5.5$, and $40 \mu \mathrm{L}$ of a DTPA-coupled derivative solution was added to the mixture. After $15 \mathrm{~min}$, the mixture was applied to a PD-10 column and eluted with $0.1 \mathrm{M}$ citrate buffer, $\mathrm{pH}$ 5.5. The derivative fractions were collected and concentrated by ultrafiltration at $4^{\circ} \mathrm{C}(16)$.

\section{Pharmacokinetic Analysis}

Iodination proteins $\left(1.0 \times 10^{8} \mathrm{cpm} / \mathrm{kg}, 0.1 \mathrm{mg} / \mathrm{kg}\right)$ in saline were injected into the tail vein of male Wistar rats. Blood $(100 \mu \mathrm{L})$ samples were periodically collected from the tail vein using a heparinized needle at $1,5,10,20,30$, and $45 \mathrm{~min}$ and at $1,2,4,8,12,18,24,36,48,60,72,84$, and $96 \mathrm{~h}$, and plasma was obtained by centrifugation. The radioactivity of plasma $(20 \mu \mathrm{L})$ samples was determined using a $\gamma$-counter (ARC-380; Aloka, Tokyo, Japan); $1 \mathrm{~mL}$ of $1 \%$ bovine serum albumin was then added to the plasma followed by $1 \mathrm{~mL}$ of $40 \%$ TCA. The sample was incubated with stirring for 10 min. After the incubation, the sample was centrifuged and the radioactivity in the pellet was recounted on the $\gamma$-counter. Urine and feces were collected at 24-h intervals, and blood was collected from other rats that were kept in a metabolic cage. The area under the plasma concentration-time curve
(AUC) was calculated by fitting an equation to the plasma precipitated by TCA concentrations of the derivatives using MULTI, a nonlinear least-squares program (17).

\section{Biodistribution Experiment}

We injected ${ }^{111}$ In-protein into the tail vein of male ddY mice. At 1, 4, 12, and $24 \mathrm{~h}$ after injection of these radiolabeled proteins, the percentage of the injected dose (\%ID) per gram or milliliter of several tissues was estimated (13). Male ddY mice received a $0.1-\mathrm{mg} / \mathrm{kg}$ dose $\left(16.7 \times 10^{6} \mathrm{cpm} / \mathrm{kg}\right)$ of ${ }^{111} \mathrm{In}$ native HSA or ${ }^{111}$ In-rHSA dimer conjugate in saline by injection into the tail vein. At 1, 4, 12, and $24 \mathrm{~h}$ after injection of these radiolabeled proteins, blood was collected from the vena cava with the animal under ether anesthesia, and plasma was obtained by centrifugation. At each of these time points, an animal was killed for excision of kidney, liver, spleen skin, muscle, and fat. These organs were then rinsed with saline and weighed, and the radioactivity contained by the samples was determined. ${ }^{111}$ In radioactivity was counted using a $\gamma$-counter (ARC-5000; Aloka).

\section{Carrageenin-Induced Paw Edema}

Paw edema was induced by the subcutaneous injection of $50 \mu \mathrm{L}$ of a saline solution containing $0.4 \%$ carrageenin into the right paw of male ddY mice, and $50 \mu \mathrm{L}$ of saline solution alone was injected to the left paw as a control. The time of carrageenin injection was designated as time 0 . After the carrageenin injection, the mice were injected with a $0.1-\mathrm{mg} / \mathrm{kg}$ dose $\left(16.7 \times 10^{6} \mathrm{cpm} / \mathrm{kg}\right)$ of ${ }^{125}$ I-native HSA or ${ }^{125} \mathrm{I}$-rHSA dimer into a tail vein at different time points. The mice were exsanguinated to death $15 \mathrm{~min}$ after the injection of radiolabeled protein. Both paws were removed, and the radioactivity in each paw was measured. Footpad thickness was measured twice immediately before the injection of carrageenin and the radiolabeled protein with a thickness
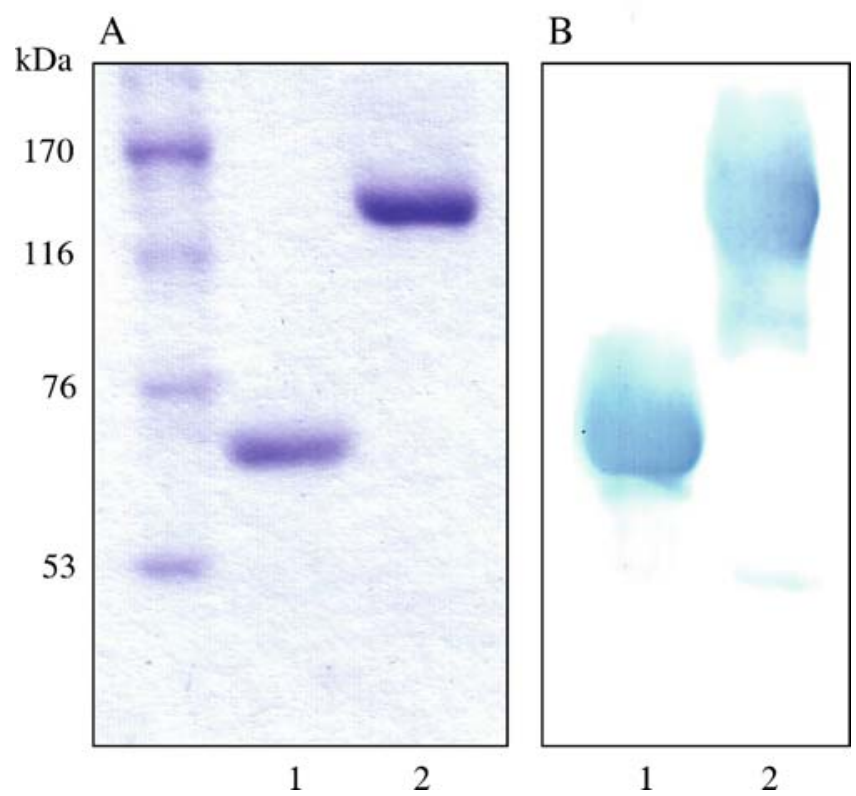

Fig. 2. Sodium dodecyl sulfate polyacrylamide gel electrophoresis (A) and Western blot analysis (B) of native HSA and rHSA dimer. Lane 1 , native HSA; lane 2 , rHSA dimer ( $3 \mu \mathrm{g}$ per lane). 


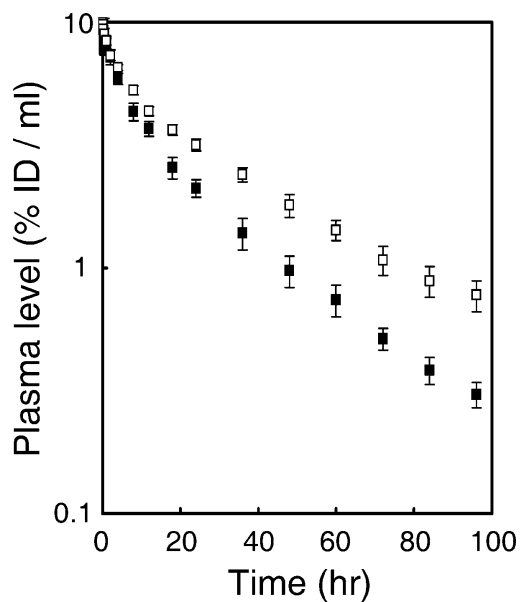

Fig. 3. Plasma level of radiolabeled proteins after intravenous administration of ${ }^{125}$ I-native HSA (filled symbol) or ${ }^{125} \mathrm{I}$-rHSA dimer (open symbol) to normal rats. Each point represents the mean $\pm \mathrm{SD}(n=4)$. \% ID, percentage of injected dose.

gage 7301 (Mitutoyo, Kanagawa, Japan). Paw edema was expressed in terms of the difference in footpad thickness between the right and the left paw.

\section{Carrageenin Air Pouch}

Inflammation of the carrageenin air pouch was induced according to the method of Tsurufuji (18). Male Wistar rats, 6 weeks of age and weighing 180-200 g, were subcutaneously injected, under light ether anesthesia, with $8.0 \mathrm{~mL}$ of air on the back to create an air pouch. After $24 \mathrm{~h}, 4.0 \mathrm{~mL}$ of a $2 \%$ (w/v) carrageenin solution in $0.9 \% \mathrm{NaCl}$ was injected into the air pouch that had been produced. The carrageenin solution was autoclaved at $120^{\circ} \mathrm{C}$ for $20 \mathrm{~min}$ and injected, after cooling, at a temperature of $40-45^{\circ} \mathrm{C}$. Immediately before the injection, penicillin and streptomycin were added in a concentration of $0.1 \mathrm{mg} / \mathrm{mL}$ each to the carrageenin solution. After 6 days of carrageenin injection, $1.0 \times 10^{8} \mathrm{cpm} / \mathrm{kg}, 0.1 \mathrm{mg} /$ $\mathrm{kg}{ }^{125}$ I-native HSA or ${ }^{125}$ I-rHSA dimer was injected into a tail vein. The day of radiolabel protein injection was designated as day 0 . Collection of blood, plasma, urine, and feces and a pharmacokinetic analysis followed the above method (Pharmacokinetic Analysis). The weight and radioactivity of the pouch fluid was measured after exsanguination of the rats to death.

\section{Statistical Analysis}

All data are presented as mean \pm SD. Significant differences between native HSA and rHSA dimer were tested by Student's $t$ test.

\section{RESULTS}

\section{Cloning and Expression of rHSA Dimer}

The rHSA dimer was expressed using $P$. pastoris. The HSA sequence contains 585 amino acids. The rHSA dimer was designed to contain two sets of the 585 amino acids fused with a linker of 10 amino acid (GGGGS) 2 . The polypeptide linker is flexible and highly hydrophilic and, hence, is resistant to proteases $(19,20)$. Construction of the expression vector of the rHSA dimer was made by a four-piece ligation (Fig. 1). The reason for the four-piece ligation was that pPIC9K cannot be used directly due to the presence of the XhoI restriction site in the kanamycin resistance gene. Furthermore, the use of other restriction enzymes would result in the addition of some other amino acids in the expressed protein. Although there is another alternative for using pPIC9, which does not contain a kanamycin resistance gene, pPIC9K is easier than pPIC9 in the selection of a highexpression strain. The N-terminal amino acid sequence of the rHSA dimer expressed in the present study was found to be consistent with that of native HSA.

\section{Structural Aspects of rHSA Dimer}

Sodium dodecyl sulfate polyacrylamide gel electrophoresis of the rHSA dimer showed a single band, and the dimer protein was recognized by an anti-HSA antibody. As shown in Fig. 2, the molecular weight of rHSA dimer ( $13 \mathrm{kDa})$ was approximately twice the molecular weight of native HSA in SDS-PAGE. The intrinsic fluorescence spectra of Trp-214, the only tryptophan residue of HSA, in the rHSA dimer was slightly low compared with that of native HSA (data not shown). To obtain information on secondary and tertiary protein structures, CD measurements were performed in the far-UV and near-UV regions (data not shown). A little decrease in the molar ellipticity of the rHSA dimer was observed in comparison with that of native HSA in the farUV CD spectrum. Although some spectral changes were observed, the near-UV CD spectra of the rHSA dimer show the same minima and shape as that of native HSA. These results suggest that the rHSA dimer structure is almost identical to that of native HSA.

\section{Pharmacokinetic Analysis of rHSA Dimer}

Pharmacokinetics of the ${ }^{125}$ I-labeled native HSA and rHSA dimer was evaluated by residual TCA-precipitable plasma radioactivity. Figure 3 shows the time courses of plasma concentration of radiolabeled rHSA dimer and native HSA, and the pharmacokinetic parameters for these two proteins using a two-compartment model are listed in Table I.

Table I. Pharmacokinetic Parameters After an Intravenous Administration of ${ }^{125}$ I-native HSA or ${ }^{125}$ I-rHSA Dimer to Normal Rats

\begin{tabular}{lcccc}
\hline & AUC $(\min \times \% \mathrm{ID} / \mathrm{mL})$ & $\mathrm{CL}_{\text {tot }}(\mu \mathrm{L} / \mathrm{h})$ & $\mathrm{Vd}_{\mathrm{ss}}(\mathrm{mL})$ & $T_{1 / 2}, \beta(\mathrm{h})$ \\
\hline${ }^{125} \mathrm{I}$-native HAS & $154.6 \pm 15.5$ & $654.6 \pm 65.0$ & $20.01 \pm 1.35$ & $26.19 \pm 0.74$ \\
${ }^{125} \mathrm{I}-\mathrm{rHSA}$ dimer & $265.6 \pm 15.2^{\dagger}$ & $380.1 \pm 25.6^{\dagger}$ & $18.13 \pm 1.36$ & $36.53 \pm 1.14^{*}$ \\
\hline
\end{tabular}

All values are mean $\pm \mathrm{SD}(n=4)$.

$* p<0.005,{ }^{\dagger} p<0.001$ vs. ${ }^{125}$ I-HSA native. 


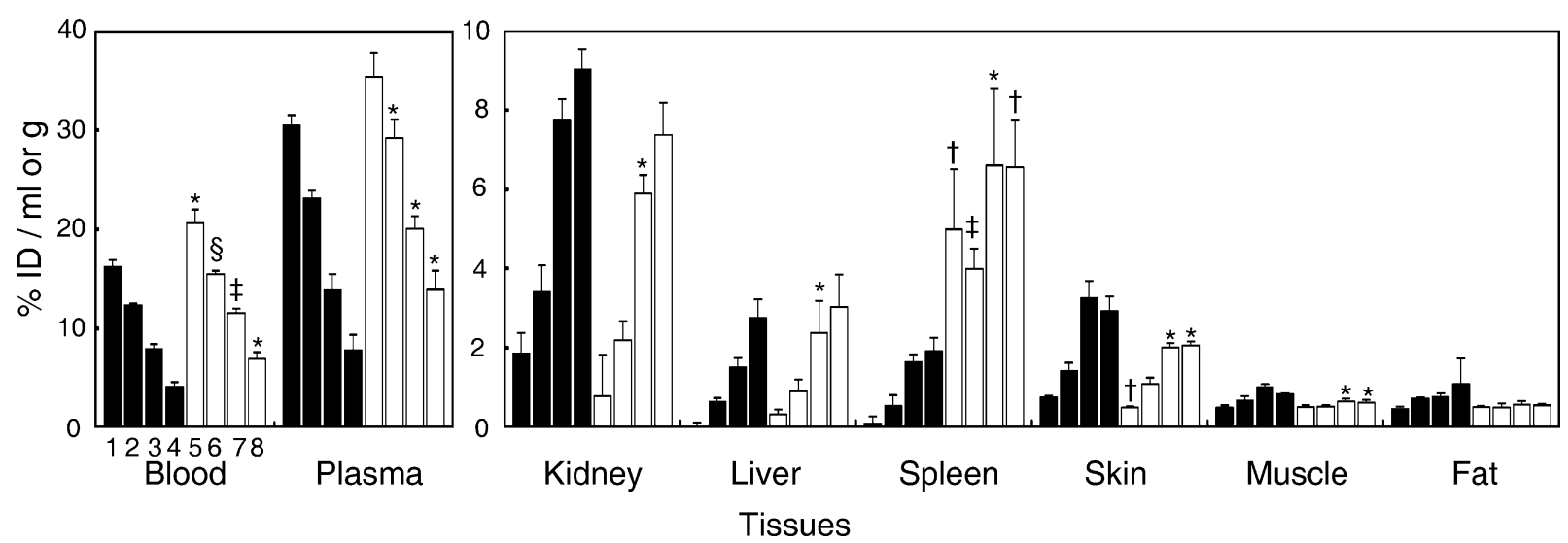

Fig. 4. Tissue distribution of ${ }^{111}$ In-native HSA (filled bar) and ${ }^{111}$ In-rHSA dimer (open bar) at 1, 4, 12, and $24 \mathrm{~h}$ after an intravenous administration to normal mice. Lanes 1 and 5, $1 \mathrm{~h}$; lanes 2 and 6, $4 \mathrm{~h}$; lanes 3 and 7, $12 \mathrm{~h}$; lanes 4 and 8, $24 \mathrm{~h}$. Each bar represents the mean \pm SD $(n=3) . * p<0.05,{ }^{\dagger} p<0.01,{ }^{*} p<0.005,{ }^{\S} p<0.001 v s .{ }^{125}$ I-native HSA.

The AUC and half-life of the ${ }^{125}$ I-rHSA dimer were increased by about 1.5 times, as compared with those of ${ }^{125}$ I-native HSA. On the other hand, the ${ }^{125}$ I-rHSA dimer showed a twothirds decrease in total body clearance $\left(\mathrm{CL}_{\text {tot }}\right)$, and a decline in the distribution volume of the steady state $\left(\mathrm{Vd}_{\mathrm{ss}}\right)$ was observed. These results suggest that the ${ }^{125}$ I-rHSA dimer has a better retention in the blood circulation, as compared with ${ }^{125}$ I-native HSA.

\section{Biodistribution of rHSA Dimer}

The tissue distribution of ${ }^{111}$ In-labeled protein was examined in the mouse (Fig. 4). The retention of the ${ }^{111} \mathrm{In}-$ rHSA dimer in the blood and plasma was confirmed when compared with the ${ }^{111}$ In-native HSA. The accumulation of the ${ }^{111}$ In-rHSA dimer in the kidney was significantly lower only after 12 and $24 \mathrm{~h}$. The accumulation of the ${ }^{111}$ In-rHSA dimer in the liver was significantly higher at $12 \mathrm{~h}$. The accumulation of the ${ }^{111}$ In-rHSA dimer in the skin, muscle, and fat was lower, with a significant decrease observed in the skin and muscle. In contrast, no significant difference was observed for the ${ }^{111}$ In-rHSA dimer in the urine and feces (Table II). The urine at 24 and $96 \mathrm{~h}$ from a mouse administered with the ${ }^{111}$ In-rHSA dimer and ${ }^{111}$ In-native HSA, respectively, was ultrafiltrated. More than $98 \%$ of the filtrate was composed of low molecular substances under 30,000 in molecular weight, and the excretion of high molecular weight protein was negligible. Ultrafiltration of the plasma containing the ${ }^{111}$ In-rHSA dimer and ${ }^{111}$ In-native HSA obtained at $1 \mathrm{~h}$ showed that more than $99 \%$ existed as high molecular weight protein.

\section{Vascular Permeability of rHSA Dimer}

The vascular permeability of the ${ }^{125} \mathrm{I}$-rHSA dimer was evaluated using carrageenin-induced mouse paw edema as a model system (Fig. 5). Carrageenin-induced mouse paw edema is known to exhibit a biphasic inflammatory response (21). In this study, the biphasic response was confirmed by measurements of the increase in the thickness of the foot. The vascular permeability of the ${ }^{125}$ I-rHSA dimer was low between 0.5 and $120 \mathrm{~h}$, especially at $0.5,1,4,8,24,48$, and $96 \mathrm{~h}$ where a significantly low permeability was observed compared with that of ${ }^{125}$ I-native HSA. These results suggest that the escape of HSA to the extravascular space during inflammation can be reduced by dimerization.

\section{Pharmacokinetic Analysis of rHSA Dimer in Carrageenin-Air-Pouch Rats}

Pharmacokinetics of ${ }^{125}$ I-labeled native HSA and the rHSA dimer in carrageenin-air-pouch rats was evaluated by measuring the residual TCA-precipitable plasma radioactivity. Table III shows the results of an analysis of the pharmacokinetic parameters using a two-compartment model. This model also showed an improvement in the retention of ${ }^{125}$ I-rHSA dimer in the blood. A significant difference in half-life of the $\beta$ phase could be observed; the AUC for the ${ }^{125} \mathrm{I}-\mathrm{rHSA}$ dimer was $11 / 2$ times larger, whereas the $\mathrm{CL}_{\text {tot }}$ value was $2 / 3$ times smaller, as compared with ${ }^{125} \mathrm{I}$ native HSA. The $\mathrm{Vd}_{\mathrm{ss}}$ for the ${ }^{125} \mathrm{I}$-rHSA dimer showed a significantly lower value than that of ${ }^{125}$ I-native HSA as well. Urine and feces were collected at 24-h intervals from rats

Table II. Urinary and Fecal Excretion of Radioactivity After an Intravenous Administration of ${ }^{111}$ In-native HSA or ${ }^{111}$ In-rHSA Dimer

\begin{tabular}{lcccr}
\hline & \multicolumn{3}{c}{ Urine (\% ID) } & \multicolumn{2}{c}{ Feces (\% ID) } \\
\cline { 2 - 4 } & $12 \mathrm{~h}$ & $24 \mathrm{~h}$ & $12 \mathrm{~h}$ & \\
\hline${ }^{111}$ In-native HAS & $1.65 \pm 0.02$ & $3.37 \pm 1.08$ & $0.82 \pm 0.15$ & $1.43 \pm 0.06$ \\
${ }^{111}$ In-rHSA dimer & $1.74 \pm 0.37$ & $2.67 \pm 0.36$ & $0.68 \pm 0.20$ & $1.32 \pm 0.18$ \\
\hline
\end{tabular}

All values are mean $\pm \mathrm{SD}(n=3)$. 


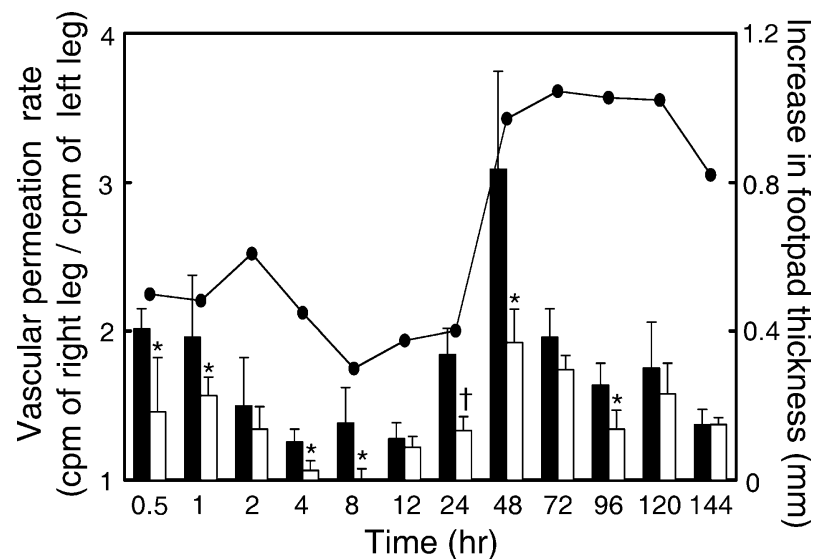

Fig. 5. Vascular permeability of ${ }^{125} \mathrm{I}$-native HSA (filled bar) and ${ }^{125} \mathrm{I}$ rHSA dimer (open bar) in mouse models of paw edema. A line graph shows the increase in footpad thickness. The data are the average values of five to seven experiments $( \pm \mathrm{SD}) .{ }^{*} p<0.05,{ }^{\dagger} p<0.01 v s$. ${ }^{125}$ I-native HSA.

different from those used for blood collection kept in metabolic cages (Table IV). According to the comparison between rats administered with the same proteins, the airpouch model rat showed a greater decrease in the early excretion in both ${ }^{125} \mathrm{I}$-native HSA and the ${ }^{125} \mathrm{I}$-rHSA dimer than normal rats. In addition, the 96-h alveolar activity of rats kept in the metabolic cage was determined. No significant difference in the weight of alveolar fluid was found between ${ }^{125}$ I-native HSA and the ${ }^{125}$ I-rHSA dimer (Table V).

\section{DISCUSSION}

Intravascular fluid therapy is a common critical care intervention. Many fluids have been studied for use in resuscitation, and these include isotonic sodium chloride solution, lactated Ringer solution, hypertonic saline, albumin, purified protein fractions, freshly frozen plasma, hetastarch, pentastarch, and dextran 70 . In spite of the many theoretical benefits of human albumin administration in critically ill patients, there has been little evidence to support its widespread clinical use. Previous systematic reviews have produced conflicting results regarding the safety and efficacy of the use of albumin $(2,22)$. The recently reported saline $v s$. albumin evaluation study has provided conclusive evidence that $4 \%$ albumin is as safe as saline for resuscitation, although no overall benefit of albumin use was reported $(3,23)$. Albumin solutions for resuscitation may still be warranted in certain highly selected patient populations such as liver transplant patients and burn patients.
Albumin plays an important role in the circulation by generating an inward oncotic force that counteracts the outward capillary hydrostatic force. Without albumin, plasma volume cannot be maintained and massive interstitial edema results. In burn shock, in which the effective circulation is reduced, albumin and extracellular fluid are rapidly shifted from the plasma and sequestered in interstitial fluids. It is commonly thought that the permeability of the capillary membranes is pathologically increased in states of shock. Burn injury is also characterized by a dramatic increase in capillary protein permeability. When shock does not develop in small burns, or is prevented by fluid therapy, the rate of protein extravasation may increase to up to 100 times the normal rate. Mechanisms that regulate plasma albumin content are the transcapillary escape rate of albumin $\left(\mathrm{TER}_{\mathrm{alb}}\right)$, lymphatic albumin return, and the albumin synthesis-to-degradation ratio. $\mathrm{TER}_{\mathrm{alb}}$ measures the whole body rate at which albumin leaves the vascular space. It is controlled by macrovascular and microvascular factors including plasma albumin concentration, plasma atrial natriuretic peptide concentration, capillary filtration coefficient, capillary hydrostatic pressure, interstitial fluid hydrostatic pressure, interstitial fluid colloid osmotic pressure, and interstitial fluid albumin concentration $(24,25)$.

Albumin leaves the circulation via several mechanisms, which vary with the tissue involved. In organs having sinusoids, i.e., liver and bone marrow, plasma can pass through large gaps in the endothelium. Some other organs have fenestrated endothelia that allow unimpeded passage; the pancreas, small intestine, and adrenal gland are examples of these. Collectively, these convection mechanisms account for about $50 \%$ of albumin transport from the circulation. The transport may be described as taking place through two sets of parallel and cylindrical pores, one set with diameters of 45-60 $\AA$ and another with a diameter of $\sim 200 \AA$. It has been shown that albumin, with an estimated radius of $35.5 \AA$, and water do not share a common pathway in crossing the endothelial monolayer, suggesting the existence of a large pore pathway for albumin (26). Results reported by Sejrsen et al. (27) indicate that the predominant transcapillary transport mechanism for ${ }^{131} \mathrm{I}$ albumin is compatible with transcapillary diffusion through pores with an effective equivalent pore radius of $145 \AA$. Hence, the extent of extravasation of albumin may be reduced by increasing its molecular size.

In this study, we attempted to express an rHSA dimer by fusing two HSA molecules, containing two sets of the protein sequence of 585 amino acids, linked with an amino acid linker (GGGGS) $)_{2}$. As can be inferred from the intrinsic fluorescence spectra and CD spectra, the structural characteristics of the rHSA dimer are almost identical to that of

Table III. Pharmacokinetic Parameters After an Intravenous Administration of ${ }^{125}$ I-native HSA or ${ }^{125}$ I-rHSA Dimer to Carrageenin-AirPouch Rats

\begin{tabular}{lclll}
\hline & $\mathrm{AUC}(\mathrm{min} \times \mathrm{mID} / \mathrm{mL})$ & $\mathrm{CL}_{\text {tot }}(\mu \mathrm{L} / \mathrm{h})$ & $\mathrm{Vd}_{\mathrm{ss}}(\mathrm{mL})$ & $\mathrm{T}$ \\
\hline${ }^{125}$ I-native HAS & $103.1 \pm 5.3$ & $974.6 \pm 53.2$ & $22.38 \pm 1.01$ & $24.85 \pm 0.55$ \\
${ }^{125}$ I-rHSA dimer & $164.0 \pm 10.1^{*}$ & $612.9 \pm 36.4^{*}$ & $19.47 \pm 1.09^{\dagger}$ & $27.30 \pm 1.40^{*}$ \\
\hline
\end{tabular}

All values are mean $\pm \mathrm{SD}(n=5)$.

${ }^{*} p<0.05,{ }^{\dagger} p<0.01,{ }^{*} p<0.001$ vs. ${ }^{125}$ I-native HSA. 
Table IV. Radioactivity in Pouch Fluid After an Intravenous Administration of ${ }^{125}$ I-native HSA or ${ }^{125}$ I-rHSA Dimer to Carrageenin-Air-Pouch Rats at $96 \mathrm{~h}$

\begin{tabular}{lcc}
\hline & Pouch fluid $(\mathrm{g})$ & Radioactivity (\% ID) \\
\hline${ }^{125}$ I-native HSA & $40.57 \pm 2.19$ & $11.12 \pm 1.60$ \\
${ }^{125}$ I-rHSA dimer & $44.10 \pm 2.30$ & $13.58 \pm 0.19$ \\
\hline
\end{tabular}

All values are mean $\pm \operatorname{SD}(n=4)$.

native HSA. The pharmacokinetic analysis of the rHSA dimer indicated that, in general, the rHSA dimer showed longer intravascular residence time, as compared with native HSA. (Fig. 3 and Table I). A biodistribution analysis of the rHSA dimer generally showed a similar tendency with that of the native HSA except for the fact that the extent of accumulation of the rHSA dimer in the spleen was much greater, whereas in the skin and muscle, the accumulation was less than that of native HSA (Fig. 4). This observation is in agreement with the findings reported by Rennen et al. (28), in which a rapid blood clearance with predominant uptake by the liver and spleen was found for the largest proteins (MW 669-800 kDa), a relatively slow blood clearance with relatively moderate uptake by the liver and spleen was seen for proteins of intermediate size (MW 66-206 kDa), and a rapid blood clearance with predominant kidney uptake was found for smaller proteins (MW 2.5-29 $\mathrm{kDa}$ ). However, it is noteworthy that the blood retention properties of the rHSA dimer were higher in comparison with native HSA (Table I). In contrast, no significant difference could be observed for the rHSA dimer and native HSA in terms of excretion in the urine and feces (Table II).

HSA is not degraded by a specific organ but by many organs such as the liver, kidney, skin, and muscle. The binding of albumin to the endothelial surface apparently initiates its transcytosis via plasmalemmal vesicles and also increases capillary permselectivity. Scavenger receptors interact with a variety of modified proteins, mediate their endocytosis and degradation, and may play an important role in protein catabolism and pathogenic processes such as atherosclerosis, aging, and diabetes. Specific albumin binding to the surface of the endothelium initiates its transcytosis across the continuous endothelium via noncoated plasmalemmal vesicles. Previous studies have identified several putative albumin-binding proteins (SPARC, gp60, gp30, and gp18). Two membrane- associated proteins, gp30 and gp18, interact more strongly with albumins that have been conformationally modified by chemical means or by surface adsorption to colloidal gold particles than with native albumin. The gp30 and gp18 proteins behave similarly to other known scavenger receptors, suggesting that gp30 and gp18 may mediate the high-affinity binding, endocytosis, and degradation of conformationally modified albumins but not native albumin, whereas albondin mediates native albumin binding, which significantly enhances its transcytosis and capillary permeability $(29,30)$.

On the other hand, the effect of an increase in vascular permeability during inflammation on the vascular permeability of the rHSA dimer was evaluated using carrageenin-induced paw edema in mice (Fig. 5). This carrageenin-induced inflammation can be used to evaluate acute exudative stage and chronic proliferative stage of carrageenin-air-pouch inflammation in rats (31). Up to $120 \mathrm{~h}$, the vascular permeability of the rHSA dimer was less than that of native HSA in the model mice. In particular, significant difference was observed during the acute inflammation stage. In addition, the pharmacokinetics of the rHSA dimer was evaluated in carrageenin-air-pouch rats (Table III). Similar profile has been obtained for the pharmacokinetics of the rHSA dimer in the inflammation mouse model as in the normal rats. In comparison with native HSA, longer blood retention and lower vascular permeability of the rHSA dimer were shown by the pharmacokinetic and vascular permeability analyses.

In contrast to our observations, McCurdy et al. (32) showed that a recombinant rabbit albumin (RSA) dimer consisting of N-terminal hexahistidinyl tagRSA(C34A) joined to G6 joined to RSA containing the $\mathrm{C} 34 \mathrm{~A}$ mutation in that order, from the $\mathrm{N}$-terminus to the $\mathrm{C}$-terminus, is more rapidly cleared in vivo than wild-type and mutant $\mathrm{C} 34 \mathrm{~A}$ rabbit albumins. The authors concluded from their study that albumin dimerization does not look promising as a tool to slow clearance. They deduced that the mechanism of the accelerated clearance of recombinant rabbit serum albumin appears to involve the reticuloendothelial system. On the other hand, Komatsu et al. (33) has demonstrated the utility of an rHSA dimer, which was cross-linked by the thiol group of Cys-34 with 1,6-bis(maleimido)hexane, and its synthetic heme hybrid as an oxygen carrier displayed a longer half-life in the bloodstream and tissue distributions that were similar to rHSA. The discrepancy between the studies may probably due to species difference, as shown in the study by Sheffield et al. (34).

Table V. Urinary and Fecal Excretion of Radioactivity (\%ID) After an Intravenous Administration of ${ }^{125}$ I-native HSA or ${ }^{125}$ I-rHSA Dimer to Normal or Carrageenin-Air-Pouch Rats

Excretion of radioactivity $(\% \mathrm{ID})$

\begin{tabular}{|c|c|c|c|c|c|c|c|c|}
\hline \multirow[b]{3}{*}{ Time (h) } & \multicolumn{4}{|c|}{${ }^{125}$ I-native HSA } & \multicolumn{4}{|c|}{${ }^{125}$ I-rHSA dimer } \\
\hline & \multicolumn{2}{|c|}{ Normal } & \multicolumn{2}{|c|}{ Air pouch } & \multicolumn{2}{|c|}{ Normal } & \multicolumn{2}{|c|}{ Air pouch } \\
\hline & Urine & Feces & Urine & Feces & Urine & Feces & Urine & Feces \\
\hline $0-24$ & $39.7 \pm 0.8$ & $1.54 \pm 0.42$ & $43.5 \pm 4.7$ & $1.58 \pm 0.54$ & $35.0 \pm 1.5$ & $1.67 \pm 0.35$ & $44.8 \pm 2.0$ & $1.51 \pm 0.41$ \\
\hline $24-48$ & $25.9 \pm 3.2$ & $1.96 \pm 0.44$ & $20.7 \pm 2.2$ & $1.69 \pm 0.36$ & $19.7 \pm 0.5$ & $1.34 \pm 0.20$ & $18.2 \pm 1.6$ & $1.22 \pm 0.12$ \\
\hline $48-72$ & $13.0 \pm 0.7$ & $1.03 \pm 0.21$ & $8.5 \pm 0.5$ & $0.54 \pm 0.15$ & $11.2 \pm 0.6$ & $0.79 \pm 0.24$ & $8.04 \pm 0.6$ & $0.53 \pm 0.13$ \\
\hline $72-96$ & $6.1 \pm 0.3$ & $0.52 \pm 0.05$ & $5.1 \pm 0.3$ & $0.40 \pm 0.01$ & $7.2 \pm 0.4$ & $0.47 \pm 0.01$ & $3.6 \pm 0.4$ & $0.26 \pm 0.02$ \\
\hline
\end{tabular}

All values are mean $\pm \operatorname{SD}(n=4)$. 
Indeed, the clearance of albumin depends on various factors including its degradation by a variety of organs, transcapillary escape, filtration and reabsorption, and lymphatic drainage, just to name a few. These pathways in turn are also influenced by the physicochemical state of the protein, such as its charge and conformation. In skin, the clearance for the most neutral modified albumins and cationic albumins was found to be 20 and $80 \%$ greater than that for native albumin, respectively. In skeletal muscle, the clearance for the most neutral modified albumins and cationic albumins was found to be 50 and $150 \%$ greater than that for native albumin, respectively. This clearly shows that charge affects the transvascular transport of albumin $(35,36)$. In this study, the fusion has been carried out through the N- and C-terminals. Since no conjugation of any of the amino acid residues has taken place, the charge of the rHSA dimer should be the same as the native HSA (monomer albumin). In addition, environmental factors such as the presence of nitric oxide have also been reported to influence the extravasation of albumin (37).

Collectively, an increase in the molecular size of HSA probably prevents or retards its convection movement through pores with diameters of 45-60 $\AA$, as observed by an increase in AUC and a decrease in the volume of distribution as well as the clearance of the dimer. Meanwhile, since the dimer exhibits structural features similar to those of native albumin, we hypothesize that transvascular movement of the rHSA dimer takes place via the same route, i.e., gp60, as evidenced by the similarity in tissue distribution profile. However, further research must be carried out to validate such speculation.

On the other hand, since HSA is an endogenous protein, it has been developed for use as a drug carrier due to its excellent biological compatibility. A promising outcome has also been reported using recombinant albumin as a synthetic heme carrier protein in producing artificial blood $(38,39)$. It is noteworthy that albumin has recently been used as a carrier to prolong the blood retention properties of therapeutic proteins such as interferon and growth hormone. Through genetic fusion technology, the therapeutic protein is fused to albumin and, as a result, the plasma half-life of the protein becomes dependent on the half-life of albumin (40-45). Fusion to albumin offers several advantages. Albumin is the most abundant protein in mammalian plasma and one of the longest lived. It lacks posttranslational modifications, with the exception of extensive disulfide bonding. If albumin can be fused inframe with a therapeutic protein as a single-chain polypeptide, the novel protein may acquire the slow clearance profile of albumin, while retaining the activity important for clinical use. On the other hand, although conjugation of polyethylene glycol with the protein may also produce similar tendency, such approach involves modification of the internal amino acid side chain, which may change the charge distribution and, hence, affect the stability of the protein. Albuferon (albumininterferon alpha) exhibits more antiviral activity at clinically achieved serum levels than standard interferon alpha or the modified interferons, Pegasys (peginterferon alpha-2a) and Peg-Intron (peginterferon alpha-2b) (43).

Clinical and preclinical results to date demonstrate that Albuferon is well tolerated, with adverse events that are transient and mostly mild to moderate in severity, and exhibits a robust antiviral activity, with a pharmacokinetic profile that supports dosing at intervals of 2 to 4 weeks. This prolongation in half-life prevents the frequent administration of the therapeutic protein. Hence, although albumin genetic fusion technology is very promising, if the blood retention properties of albumin can be improved further, it is expected that such albumin will be of great clinical use as a new drug delivery system material in addition to being a plasma expander.

\section{ACKNOWLEDGMENTS}

We wish to thank the members of the Gene Technology Center in Kumamoto University for their important contributions to these experiments.

\section{REFERENCES}

1. T. Peters. All About Albumin: Biochemistry, Genetics, and Medical Applications, Academic, San Diego, 1996.

2. Cochrane Injuries Group. Human albumin administration in critically ill patients: systematic review of randomised controlled trials. Cochrane Injuries Group Albumin Reviewers. BMJ 317: 235-240 (1998).

3. S. Finfer, R. Bellomo, N. Boyce, J. French, J. Myburgh, and R. Norton. A comparison of albumin and saline for fluid resuscitation in the intensive care unit. N. Engl. J. Med. 350:2247-2256 (2004).

4. H. Rubin, S. Carlson, M. DeMeo, D. Ganger, and R. M. Craig. Randomized, double-blind study of intravenous human albumin in hypoalbuminemic patients receiving total parenteral nutrition. Crit. Care Med. 25:249-252 (1997).

5. G. Akerstrom and B. Lisander. Tissue extravasation of albumin from intraabdominal trauma in rats. Acta Anaesthesiol. Scand. 35:257-261 (1991).

6. M. P. Margarson and N. C. Soni. Tissue extravasation of albumin from intraabdominal trauma in rats. Br. J. Anaesth. 92:821-826 (2004).

7. S. Berg, M. Golster, and B. Lisander. Albumin extravasation and tissue washout of hyaluronan after plasma volume expansion with crystalloid or hypooncotic colloid solutions. Acta Anaesthesiol. Scand. 46:166-172 (2002).

8. M. Mathru, B. Blakeman, D. J. Dries, B. Kleinman, and P. Kumar. Permeability pulmonary edema following lung resection. Chest 98:1216-1218 (1990).

9. K. Byrne, J. L. Tatum, D. A. Henry, J. I. Hirsch, M. Crossland, T. Barnes, J. A. Thompson, J. Young, and H. J. Sugerman. Increased morbidity with increased pulmonary albumin flux in sepsis-related adult respiratory distress syndrome. Crit. Care Med. 20:28-34 (1992)

10. S. Galatius, L. Bent-Hansen, H. Wroblewski, V. B. Sorensen, T. Norgaard, and J. Kastrup. Plasma disappearance of albumin and impact of capillary thickness in idiopathic dilated cardiomyopathy and after heart transplantation. Circulation 102:319-325 (2000).

11. T. Oshima, F. S. Laroux, L. L. Coe, Z. Morise, S. Kawachi, P. Bauer, M. B. Grisham, R. D. Specian, P. Carter, S. Jennings, D. N. Granger, T. Joh, and J. S. Alexander. Interferon-gamma and interleukin-10 reciprocally regulate endothelial junction integrity and barrier function. Microvasc. Res. 61:130-143 (2001).

12. R. F. Chen. Removal of fatty acids from serum albumin by charcoal treatment. J. Biol. Chem. 242:173-181 (1967).

13. S. Matsushita, Y. Isima, V. T. Chuang, H. Watanabe, S. Tanase, T. Maruyama, and M. Otagiri. Functional analysis of recombinant human serum albumin domains for pharmaceutical applications. Pharm. Res. 21:1924-1932 (2004).

14. J. Bhatia, S. K. Sharma, K. A. Chester, R. B. Pedley, R. W. Boden, D. A. Read, G. M. Boxer, N. P. Michael, and R. H. Begent. Catalytic activity of an in vivo tumor targeted anti-CEA scFv::carboxypeptidase G2 fusion protein. Int. J. Cancer 85:571-577 (2000) 
15. D. J. Hnatowich, W. W. Layne, and R. L. Childs. The preparation and labeling of DTPA-coupled albumin. Int. J. Appl. Radiat. Isot. 33:327-332 (1982).

16. H. Katsumi, M. Nishikawa, S. F. Ma, F. Yamashita, and M. Hashida. Physicochemical, tissue distribution, and vasodilation characteristics of nitrosated serum albumin: delivery of nitric oxide in vivo. J. Pharm. Sci. 93:2343-2352 (2004).

17. K. Yamaoka, Y. Tanigawara, T. Nakagawa, and T. A. Uno. Pharmacokinetic analysis program (multi) for microcomputer. $J$. Pharmacobio-dyn. 4:879-885 (1981).

18. S. Tsurufuji, H. Sato, K. R. Min, and K. Ohuchi. Difference in the anti-inflammatory effect of indomethacin between acute and chronic stages of carrageenin-induced inflammation. J. Pharmacobio-dyn. 1:8-14 (1978).

19. D. Shan, O. W. Press, T. T. Tsu, M. S. Hayden, and J. A. Ledbetter. Characterization of scFv-Ig constructs generated from the anti-CD20 mAb 1F5 using linker peptides of varying lengths. J. Immunol. 162:6589-6595 (1999).

20. M. Gustavsson, J. Lehtio, S. Denman, T. T. Teeri, K. Hult, and M. Martinelle. Stable linker peptides for a cellulose-binding domain-lipase fusion protein expressed in Pichia pastoris. Protein Eng. 14:711-715 (2001).

21. I. Posadas, M. Bucci, F. Roviezzo, A. Rossi, L. Parente, L. Sautebin, and G. Cirino. Carrageenan-induced mouse paw oedema is biphasic, age-weight dependent and displays differential nitric oxide cyclooxygenase-2 expression. Br. J. Pharmacol. 142:331-338 (2004).

22. M. M. Wilkes and R. J. Navickis. Patient survival after human albumin administration. A meta-analysis of randomized, controlled trials. Ann. Intern. Med. 135:149-164 (2001).

23. E. Fan and T. E. Stewart. Albumin in critical care: SAFE, but worth its salt? Crit. Care 8:297-299 (2004).

24. B. Rippe and B. Haraldsson. Transport of macromolecules across microvascular walls: the two-pore theory. Physiol. Rev. 74:163-219 (1994).

25. C. C. Michel and F. E. Curry. Microvascular permeability. Physiol. Rev. 79:703-761 (1999).

26. R. O. Dull, H. Jo, H. Sill, T. M. Hollis, and J. M. Tarbell. The effect of varying albumin concentration and hydrostatic pressure on hydraulic conductivity and albumin permeability of cultured endothelial monolayers. Microvasc. Res. 41:390-407 (1991).

27. P. Sejrsen, W. P. Paaske, and O. Henriksen. Capillary permeability of 131I-albumin in skeletal muscle. Microvasc. Res. 29: 265-281 (1985).

28. H. J. Rennen, J. Makarewicz, W. J. Oyen, P. Laverman, F. H. Corstens, and O. C. Boerman. The effect of molecular weight on nonspecific accumulation of $(99 \mathrm{~m}) \mathrm{T}$-labeled proteins in inflammatory foci. Nucl. Med. Biol. 28:401-408 (2001).

29. J. E. Schnitzer, A. Sung, R. Horvat, and J. Bravo. Preferential interaction of albumin-binding proteins, gp30 and gp18, with conformationally modified albumins. Presence in many cells and tissues with a possible role in catabolism. J. Biol. Chem. 267:24544-24553 (1992).

30. J. E. Schnitzer and P. Oh. Albondin-mediated capillary permeability to albumin. Differential role of receptors in endothelial transcytosis and endocytosis of native and modified albumins. $J$. Biol. Chem. 269:6072-6082 (1994).

31. H. Sato, M. Hashimoto, K. Sugio, K. Ohuchi, and S. Tsurufuji. Comparative study between steroidal and nonsteroidal antiinflammatory drugs on the mode of their actions on vascular permeability in rat carrageenin-air-pouch inflammation. $J$. Pharmacobio-dyn. 3:345-352 (1980).
32. T. R. McCurdy, S. Gataiance, L. J. Eltringham-Smith, and W. P. Sheffield. A covalently linked recombinant albumin dimer is more rapidly cleared in vivo than are wild-type and mutant C34A albumin. J. Lab. Clin. Med. 143:115-124 (2004).

33. T. Komatsu, Y. Oguro, Y. Teramura, S. Takeoka, J. Okai, M. Anraku, M. Otagiri, and E. Tsuchida. Physicochemical characterization of cross-linked human serum albumin dimer and its synthetic heme hybrid as an oxygen carrier. Biochim. Biophys. Acta 1675:21-31 (2004).

34. W. P. Sheffield, A. Mamdani, G. Hortelano, S. Gataiance, L. Eltringham-Smith, M. E. Begbie, R. A. Leyva, P. S. Liaw, and F. A Ofosu. Effects of genetic fusion of factor IX to albumin on in vivo clearance in mice and rabbits. Br. J. Haematol. 126:565-573 (2004)

35. R. R. Gandhi and D. R. Bell. Importance of charge on transvascular albumin transport in skin and skeletal muscle. Am. J. Physiol. 262:H999-H1008 (1992).

36. H. Vink and B. R. Duling. Capillary endothelial surface layer selectively reduces plasma solute distribution volume. Am. J. Physiol, Heart Circ. Physiol. 278:H285-H289 (2000).

37. L. W. Chen, J. S. Wang, B. Hwang, J. S. Chen, and C. M. Hsu. Reversal of the effect of albumin on gut barrier function in burn by the inhibition of inducible isoform of nitric oxide synthase. Arch. Surg. 138:1219-1225 (2003).

38. E. Tsuchida, K. Ando, H. Maejima, N. Kawai, T. Komatsu, S. Takeoka, and H. Nishide. Properties of and oxygen binding by albumin-tetraphenylporphyrinatoiron(II) derivative complexes. Bioconjug. Chem. 8:534-538 (1997).

39. T. Komatsu, H. Yamamoto, Y. Huang, H. Horinouchi, K. Kobayashi, and E. Tsuchida. Exchange transfusion with synthetic oxygen-carrying plasma protein -albumin-heme- into an acute anemia rat model after seventy-percent hemodilution. $J$. Biomed. Mater. Res., A. 71:644-651 (2004).

40. S. Syed, P. Schuyler, M. Kulczycky, and W. P. Sheffield. Potent antithrombin activity and delayed clearance from the circulation characterize recombinant hirudin genetically fused to albumin. Blood 89:3243-3252 (1997).

41. P. Yeh, D. Landais, M. Lemaitre, I. Maury, J.-Y. Crenne, J. Becquart, A. Murry-Brelier, F. Boucher, G. Montay, R. Fleer, P.-H. Hirel, J.-F. Mayaux, and D. Klatzmann. Design of yeastsecreted albumin derivatives for human therapy: biological and antiviral properties of a serum albumin-CD4 genetic conjugate. Proc. Natl. Acad. Sci. U. S. A. 89:1904-1908 (1992).

42. M. S. Dennis, M. Zhang, Y. G. Meng, M. Kadkhodayan, D. Kirchhofer, D. Combs, and L. A. Damico. Albumin binding as a general strategy for improving the pharmacokinetics of proteins. J. Biol. Chem. 277:35035-35043 (2002).

43. B. L. Osborn, H. S. Olsen, B. Nardelli, J. H. Murray, J. X. Zhou, A. Garcia, G. Moody, L. S. Zaritskaya, and C. Sung. Pharmacokinetic and pharmacodynamic studies of a human serum albumin-interferon-alpha fusion protein in cynomolgus monkeys. J. Pharmacol. Exp. Ther. 303:540-548 (2002).

44. C. Sung, B. Nardelli, D. W. LaFleur, E. Blatter, M. Corcoran, H. S. Olsen, C. E. Birse, O. K. Pickeral, J. Zhang, D. Shah, G Moody, S. Gentz, L. Beebe, and P. A. Moore. An IFNbeta-albumin fusion protein that displays improved pharmacokinetic and pharmacodynamic properties in nonhuman primates. J. Interferon Cytokine Res. 23:25-36 (2003).

45. B. L. Osborn, L. Sekut, M. Corcoran, C. Poortman, B. Sturm, G. Chen, D. Mather, H. L. Lin, and T. J. Parry. Albutropin: a growth hormone-albumin fusion with improved pharmacokinetics and pharmacodynamics in rats and monkeys. Eur. J. Pharmacol. 456:149-158 (2002). 